\title{
Treatment of left main coronary artery stenosis with the STENTYS self-expandable drug-eluting stent - a pilot registry
}

\author{
Grzegorz Smolka ${ }^{1}$, Wojciech Wańha ${ }^{1}$, Tomasz Roleder ${ }^{2}$, Aleksandra Pluta ${ }^{3}$, Andrzej Ochała ${ }^{1}$ \\ ${ }^{1} 3^{\text {rd }}$ Department of Cardiology, Medical University of Silesia, Katowice, Poland \\ ${ }^{2}$ Department of Cardiology, Medical University of Silesia, Katowice, Poland \\ ${ }^{3}$ Medical University of Silesia, Katowice, Poland
}

Postep Kardiol Inter 2014; 10, 4 (38): 226-230

DOI: $10.5114 /$ pwki.2014.46941

\begin{abstract}
A bstract
Percutaneous coronary intervention (PCI) of the left main coronary artery (LMCA) for revascularization after stenosis is still considered controversial therapy. Previous studies were performed with balloon-expandable drug-eluting stents (DES). Balloon-expandable stents presented a challenge because they were not able to adapt effectively to variation in the vessel lumen. There are limited data on LMCA therapy with self-expandable DES for treatment of medial and distal lesions. The advantages of a self-apposing stent are adaptation to vessel size, vessel tapering, stent sizing, and good apposition. This was a pilot study to determine safety and device success rate in patients with middle and distal LMCA stenosis treated with the STENTYS self-expanding coronary DES stent. The primary endpoints were device success, acute procedural success and in-hospital and 30-day MACE. Twenty-four patients were included. Median logistic EuroSCORE was 1.6\% (1.1-2.6\%). Median Syntax score was 20.0 (20.0-27.2) points. Significant stenosis according to the anatomical region was in the middle of the LMCA in 5 cases $(21 \%)$ and the distal part in 19 (79\%). Stent sizes used were: $3.0 \times 3.5 \mathrm{~mm}$ in $9(37.5 \%) ; 3.5 \times 4.0 \mathrm{~mm}$ in $3(12.5 \%) ; 3.5 \times 4.5 \mathrm{~mm}$ in $12(50 \%)$. Device success and acute procedural success were achieved in 23 patients (95.8\%), with no edge dissection in any patient. In 1 patient the proximal end of the stent protruded into the aorta. In all patients during their hospitalization and 30-day follow-up there were no adverse events. The data compiled from this small, single-center pilot study suggest that the STENTYS self-expanding coronary stent may be a reasonable approach to treat lesions within the LMCA. These results warrant a larger future clinical trial.
\end{abstract}

Key words: left main, percutaneous coronary intervention, self-expanding stent.

\section{Introduction}

Percutaneous coronary intervention $(\mathrm{PCl})$ of the left main coronary artery (LMCA) for revascularization after stenosis is still considered controversial therapy. The LMCA supplies significant blood flow to the myocardium. In the presence of severe LMCA disease, patients are placed at higher risk for arrhythmias or left ventricular dysfunction. Immediate, effective therapy is warranted which would ideally provide optimal care to the patient to relieve symptoms, revascularization and reperfusion supplied to the damaged myocardium with minimal adverse consequences. Location of the lesion or stenosis plays a critical role in determining the best therapy for optimal outcome. The LMCA is divided into three anatomic regions: the ostium, which is the origin of the
LMCA from the aorta; a mid/medial-portion; and a distal portion. The LMCA then bifurcates into the left anterior descending (LAD) and left circumflex (LCX) arteries. These vessels vary in size and characteristics.

While the PCI technique has become somewhat more favorable in recent years, there still remain procedural challenges. Based on available evidence in lesions localized to certain areas, $\mathrm{PCl}$ is favored over coronary artery bypass grafting (CABG) as the better option for revascularization, in those patients who exhibited low PCI procedural risk or high surgical complication risk. It should be noted that previously CABG was considered the gold standard treatment for revascularization. The European Society of Cardiology current guidelines consider the presence of an LMCA middle lesion as a class Ila indication for $\mathrm{PCl}$, level of evidence $\mathrm{B}$, and distal LMCA bifur-

\section{Corresponding author:}

Grzegorz Smolka MD, PhD, $3^{\text {rd }}$ Department of Cardiology, Medical University of Silesia, 45-47 Ziołowa St, 40-635 Katowice, Poland, phone: +48 501201622 , e-mail: grsm18@wp.pl

Received: 29.08.2014, accepted: 12.11.2014. 
cation as a class Ilb indication for $\mathrm{PCl}$, level of evidence $B$ [1]. Level of evidence $B$ indicates that there was a lack of data derived from multiple randomized clinical trials or meta-analyses when the guidelines were established. Capodanno et al. [2] reviewed data from several randomized clinical trials, which has provided greater clarity between the benefits or risk of these two types of therapy. Their results indicated that $\mathrm{PCl}$ was associated with a non-significantly higher 1-year rate of major adverse cerebrocardiovascular events (MACCE) compared with CABG. The MACCE is defined as death, myocardial infarction (MI), target vessel revascularization (TVR), or stroke. However, their results also indicated that there were no significant differences in deaths or MIs and there were fewer strokes when PCI therapy was utilized. While their analysis provided more detailed information, with fewer limitations than previously reported by some research studies, there continue to be questions that only future research studies can address. These questions center on what beneficial improvements could the $\mathrm{PCl}$ procedure offer if the focus was placed on the type of stent, the stent's properties, sizes and vessel characteristics used for revascularization. These aspects seem to create most of the challenges preventing a successful patient outcome with $\mathrm{PCl}$ therapy.

\section{Aim}

This study is an analysis of revascularization of the medial or distal portion of LMCA lesions with a self-expendable drug-eluting stent (DES).

\section{Material and methods}

With this focus in mind, we conducted a one-center, retrospective, non-randomized, single-arm pilot study. Inclusion criteria were: significant stenosis of the distal LMCA, lesion length < $25 \mathrm{~mm}$ (LMCA and main branch), side branch (SB) without significant stenosis involving more than 5 proximal $\mathrm{mm}$, and the patient had to have been disqualified from surgical treatment by a heart team. Exclusion criteria were: any lesion involving the ostial part of the LMCA, reference diameter of $L M>4.5 \mathrm{~mm}$, and when the distal reference diameter of the main branch was too big or too small to use a STENTYS DES stent. Planned $\mathrm{PCl}$ of another segment occurred during a 6-month period.

Primary endpoints were:

1. Device success - defined as ability to implant the stent in the target lesion, including complete coverage of the target lesion, but the stent could not protrude outside an established location, including protrusion into the aorta. Also, the stent had to achieve an optimal angiographic result.

2. Acute procedural success - defined as device success without MACCE (defined as myocardial infarction, cardiovascular death and stroke) during the immediate $72 \mathrm{~h}$ after $\mathrm{PCl}$.
3. Number of MACCE in 30-day follow-up.

An optimal result was defined as $<10 \%$ residual stenosis of the target lesion, without angiographically visible dissection at the edges of the stent, and/or the SB with TIMI 3 flow in both vessels post-procedure. Myocardial infarction was defined according to the ESC third universal definition of myocardial infarction [3]. Target lesion revascularization (TLR) was defined as a repeat treatment of a lesion located within the index coronary artery segment. MACCE were reviewed periprocedurally, at discharge and after 30 days of follow-up.

\section{Study procedure}

All study patients' therapy was discussed with the heart team. Those that met our inclusion criteria were enrolled. They had critical stenosis of the medial and distal LMCA. The ostium of the LMCA had to be free of atherosclerosis. Significant LMCA stenosis was defined as angiographic stenosis of $\geq 50 \%$. Intravascular ultrasound (IVUS) assessment was performed according to daily practice, but it was mandatory in the case of stenosis between $50 \%$ and $80 \%$ on angiography. Minimal luminal area (MLA) below $6 \mathrm{~mm}^{2}$ was considered as critical according to the outcome from the LITRO study [4]. The principle of the stent implantation was to cover the whole lesion length using one STENTYS stent. Disconnecting struts for side-branch access was done if the diameter of the side branch was $>2.0 \mathrm{~mm}$ with TIMI flow $<3$ and/or stenosis > 50\%. Post-dilatation of the LMCA was performed in all patients. A second stent for the side branch was implanted when the result of balloon angioplasty was not optimal, especially in cases of: heavily calcified and/or severely angulated entry, and/or lesion length $>5 \mathrm{~mm}$, and/or other significant disease requiring treatment. Before the procedure, patients received dual antiplatelet therapy (DAPT): aspirin (75 to $150 \mathrm{mg}$ orally indefinitely) and clopidogrel $75 \mathrm{mg} /$ day for 12 months. To achieve activated coagulation time (ACT) > $300 \mathrm{~s}$ during the procedure, intravenous unfractionated heparin (70$100 \mathrm{lU} / \mathrm{kg}$ ) was administered. The vascular approach depended on the operator's decision.

\section{Results}

\section{Demographic and lesion characterization}

Between February 2012 and March 2014, 24 patients were included in this study. Median age was 64.5 (95\% Cl: 58.0-68.2) years. Patients' characteristics, risk factors and clinical presentation are listed in Table I. There were $83 \%$ females and $17 \%$ males.

The coronary issues were stable CAD $n=6(25 \%)$; unstable angina $n=16$ (67\%); NSTEMI $n=2(8 \%)$. Within this group, 4 (17\%) patients had diabetes, 21 (87\%) had hypertension, 2 (8\%) had chronic kidney disease, 9 (37\%) had a history of $\mathrm{MI}$, and 10 (41\%) patients had previous CABG. Reasons given for not selecting CABG as therapy 
Table I. Demographic and clinical data $(n=24)$

\begin{tabular}{lc}
\hline Clinical characteristics & Results \\
\hline Age, median (IQR) [year] & $64.5(95 \%$ Cl: $58.0-68.2)$ \\
\hline Female, $n(\%)$ & $20(83)$ \\
\hline \begin{tabular}{l} 
Discharge diagnosis, $n(\%):$ \\
\hline Stable CAD
\end{tabular} \\
\hline UA & $6(25)$ \\
\hline NSTEMI & $16(67)$ \\
\hline STEMI & $2(8)$ \\
\hline Post-CABG & $10(41)$ \\
\hline Diabetes mellitus, $n$ (\%) & $4(17)$ \\
\hline Hypertension, $n(\%)$ & $21(87)$ \\
\hline LVEF, median (95\% CI) & $50(43.7-55.0)$ \\
\hline Previous MI, $n$ (\%) & $9(37)$ \\
\hline CKD, $n$ (\%) & $2(8)$ \\
\hline EuroSCORE logistic STS score, median \\
(IQR) [\%]
\end{tabular}

were previous cardiac surgery, high risks due to comorbidities and patient disagreement.

Left ventricle ejection fraction (LVEF) values were recorded in all patients, and the median LVEF was 50 (95\% Cl: 43.7-55.0)\%. Median logistic EuroSCORE was $1.6 \%$ (95\% Cl: 1.1-2.6). Coronary angiography (Table II) revealed a Syntax score of 20.0 points (95\% Cl: 20.0 27.2). In 18 (75\%) cases $\mathrm{PCI}$ was performed from a radial vessel approach. The $\mathrm{PCl}$ was mostly performed using $6 \mathrm{Fr}$ guiding catheters. In the case of Medina classification 1-1-1, 7 Fr guiding catheters were used. The PCI in Medina classification 1-1-1 was performed with two guidewires, because it was not possible to put two balloons in a $6 \mathrm{Fr}$ catheter. According to the Medina classification, 11 (45\%) patients had 1-1-1 and 13 (54\%) had the side branch free of disease (1-0-1). The median LMCA reference diameter was $4.0 \mathrm{~mm}$ (QCA) (95\% Cl: 3.854.15), and median stenosis assessed using QCA was 70 (60.0-80.0). The length of stenosis was 23 (20-25). Stent sizes used were: $3.5 \times 4.5 \mathrm{~mm}$ in 12 cases (50\%), $3.5 \times$ $4.0 \mathrm{~mm}$ in 3 cases $(12.5 \%)$ and $3.0 \times 3.5 \mathrm{~mm}$ in 9 cases (37.5\%). In most cases (20.83\%) a self-expanding stent was implanted in the sequence LMCA to LAD. In all cases, the target lesion was fully covered, and it was not necessary to implant an additional stent at the proximal or distal edge of the STENTYS stent. In one case there was excessive protrusion of the stent into the lumen of the aorta (device failure). So the device was successful in 23 (95.8\%) cases. In all cases, post-dilatation was performed. Strut disconnection for SB access was done in 18 patients (75\%), mostly because of angiographically
Table II. Angiographic and procedural data $(n=24)$

\begin{tabular}{|c|c|}
\hline Angiographic characteristics & Results \\
\hline SYNTAX score, median (IQR) [point] & $20.0(20.0-27.2)$ \\
\hline Radial approach, $n(\%)$ & $18(75)$ \\
\hline Stenosis assessed using QCA, median $(95 \% \mathrm{CI})$ & $70(60.0-80.0)$ \\
\hline Length of stenosis, median ( $95 \% \mathrm{Cl}$ ) & $23(20.0-25.0)$ \\
\hline Residual stenosis, median $(95 \% \mathrm{Cl})$ & $5.1(3.0-7.2)$ \\
\hline \multicolumn{2}{|l|}{ LMCA lesion location, $n(\%)$ : } \\
\hline Middle part & $5(21)$ \\
\hline Distal part & $19(79)$ \\
\hline \multicolumn{2}{|l|}{ Medina classification, $n(\%)$ : } \\
\hline $1-1-1$ & $11(45)$ \\
\hline $1-0-1$ & $13(54)$ \\
\hline \multicolumn{2}{|l|}{ Number of stents in different lengths, $n(\%)[\mathrm{mm}]$ : } \\
\hline 22 & $12(51)$ \\
\hline 23 & $1(4)$ \\
\hline 27 & $11(45)$ \\
\hline \multicolumn{2}{|c|}{ Number of stents in different diameters, $n(\%)[\mathrm{mm}]$ : } \\
\hline $3.0-3.5$ & $9(37.5)$ \\
\hline $3.5-4.0$ & $3(12.5)$ \\
\hline $3.5-4.5$ & $12(50)$ \\
\hline Post-dilatation, $n$ (\%) & $24(100)$ \\
\hline Strut disconnection for SB, $n(\%)$ & $18(75)$ \\
\hline Balloon-expandable stent for SB, $n(\%)$ & $4(16)$ \\
\hline Device success, $n$ (\%) & $23(96)$ \\
\hline
\end{tabular}

critical stenosis of the SB (15 cases) and TIMI flow $<3$ in 3 cases. For 4 (16\%) patients a second (balloon-expandable) stent for the SB was necessary because of significant stenosis of the ostium and proximal segment of the SB. There were no adverse events in the perioperative period. The acute procedural success rate was $95.8 \%$. In all patients during the index hospitalization and 30-day follow-up there were no adverse events.

\section{Discussion}

The gold standard of therapy for patients with LMCA disease has been CABG. Because of patient complexity, vessel attributes/differences, and surgical risks, there was a need to evaluate other treatment options. Research studies utilizing $\mathrm{PCl}$ with stent placement demonstrated the feasibility and safety of this therapy, as an alternative option. However, treating LMCA stenosis via a $\mathrm{PCl}$ approach remains a challenge for some interven- 
tional cardiologists. Complications after this therapy, as well as the therapy itself, seems to be the focal concern. Different types of stents have been used such as bare metal or drug-eluting stents, using a balloon expandable technique for vessel placement. Balloon expandable stents presented a challenge because they were not able to adapt effectively to variation in the vessel lumen [5-7]. Data from some studies illustrated increased need for repeat revascularization with $\mathrm{PCl}$ vs. CABG in patients with LMCA stenosis but no difference in mortality or combined rates of death and MI [7-10]. Significant advances with the new generation of stents have improved the $\mathrm{PCl}$ outcome in patients, but more advances in this technique are needed. In an aging population with a high prevalence of atherosclerosis, recurrent ischemia and acute coronary syndrome (ACS), LMCA stenosis could become a more frequent clinical problem.

Lesion location is a key factor contributing to the restenosis of a vessel. At least $50 \%$ to $60 \%$ of unprotected LMCA lesions involve the distal bifurcation, often with significant involvement of the ostium of both distal branches [11]. Bifurcation lesions require a complex stenting approach, which has been an independent factor for stent thrombosis [12, 13]. The STENTYS stent covers the carina during a provisional approach and could be the best solution to avoid stent thrombosis [14].

In this study, we successfully treated middle and distal LMCA lesions with a self-expanding coronary DES. The STENTYS DES coronary stent is a self-expanding, nitinol, paclitaxel-eluting $\left(0.8 \mu \mathrm{g} / \mathrm{mm}^{2}\right.$ stent $)$ stent incorporated in ProTeqtor (Hemoteq AG, Würselen, Germany), a durable polymer matrix of polysulfone (PSU) and soluble polyvinylpyrrolidone (PVP) that acts as an excipient. This stent is deployed by retracting a sheath with a nominal strut width of 0.0027 " (68 microns). A 6 Fr compatible, rapid-exchange delivery system delivers the stent into position over a conventional 0.014" guidewire. The STENTYS DES stents are available in different sizes, small $(2.5-3.0 \mathrm{~mm})$, medium (3.0-3.5 mm) and large (3.5-4.5 mm), and in lengths $17 \mathrm{~mm}, 22 \mathrm{~mm}$ and $27 \mathrm{~mm}$, respectively. The stent has a Z-shaped design that is linked together by small interconnections, which can be disconnected by balloon inflation between the struts to create side branch access, if needed. The STENTYS DES is covered with a retractable sheath and has three markers: proximal, distal, and outer sheath. When implanting this device, the outer sheath marker should be located at least $5 \mathrm{~mm}$ distally to the lesion in order to achieve full coverage. Another advantage of this stent is simple disconnection of the stent struts and anatomical reconstruction of the bifurcation shape [15]. A major benefit of using the self-expandable STENTYS stent is its ability to optimize the apposition of the stent to the vessel wall. Other advantages of the STENTYS stent are easy implantation and easy access to the side branch.
Intravascular ultrasound (IVUS) guidance is recommended to evaluate the severity of a lesion in the LMCA [4] and to optimize angioplasty. Furthermore, according to the MAIN-COMPARE registry, $\mathrm{PCl}$ of the unprotected LMCA with IVUS assessment is associated with a mortality reduction [16]. However, in this study only 6 (6) patients had PCI with IVUS assessment because of borderline stenosis. A 30-day post-procedural follow-up demonstrated no MACCE events in this population.

Limitations of STENTYS stent:

- Does not provide coverage of ostium of LMCA.

- Available only as paclitaxel-eluting stents.

- Currently not suitable for larger diameter of LMCA over $4.5 \mathrm{~mm}$.

Limitations of this study: 1) this was a pilot study, so the population was small; 2) there was no randomization of patients as to type of stenting technique (self-expanding or balloon-expandable).

\section{Conclusions}

This study evaluated the safety and feasibility of the STENTYS DES self-expanding coronary stent in middle and distal LMCA stenosis. The data compiled from this small, single-center pilot study suggest that the STENTYS DES self-expanding coronary stent may be a reasonable approach to treating medial and distal LMCA lesions.

\section{References}

1. Task Force on Myocardial Revascularization of the European Society of C, the European Association for Cardio-Thoracic S, European Association for Percutaneous Cardiovascular I, et al. Guidelines on myocardial revascularization. Eur J Cardiothorac Surg 2010; 38 Suppl.: S1-52.

2. Capodanno D, Stone GW, Morice MC, et al. Percutaneous coronary intervention versus coronary artery bypass graft surgery in left main coronary artery disease: a meta-analysis of randomized clinical data. J Am Coll Cardiol 2011; 58: 1426-32.

3. Thygesen K, Alpert JS, Jaffe AS, et al. Third universal definition of myocardial infarction. Circulation 2012; 126: 2020-35.

4. de la Torre Hernandez JM, Hernandez Hernandez F, Alfonso F, et al. Prospective application of pre-defined intravascular ultrasound criteria for assessment of intermediate left main coronary artery lesions results from the multicenter LITRO study. J Am Coll Cardiol 2011; 58: 351-8.

5. Buszman PE, Buszman PP, Kiesz RS, et al. Early and long-term results of unprotected left main coronary artery stenting: the LE MANS (Left Main Coronary Artery Stenting) registry. J Am Coll Cardiol 2009; 54: 1500-11.

6. Morice MC, Serruys PW, Kappetein AP, et al. Outcomes in patients with de novo left main disease treated with either percutaneous coronary intervention using paclitaxel-eluting stents or coronary artery bypass graft treatment in the Synergy Between Percutaneous Coronary Intervention with TAXUS and Cardiac Surgery (SYNTAX) trial. Circulation 2010; 121: 2645-53.

7. Boudriot E, Thiele H, Walther T, et al. Randomized comparison of percutaneous coronary intervention with sirolimus-eluting stents versus coronary artery bypass grafting in unprotected left main stem stenosis. J Am Coll Cardiol 2011; 57: 538-45. 
8. Park SJ, Kim YH, Park DW, et al. Randomized trial of stents versus bypass surgery for left main coronary artery disease. N Engl J Med 2011; 364: 1718-27.

9. Chieffo A, Magni V, Latib A, et al. 5-year outcomes following percutaneous coronary intervention with drug-eluting stent implantation versus coronary artery bypass graft for unprotected left main coronary artery lesions the Milan experience. JACC Cardiovasc Interv 2010; 3: 595-601.

10. Mohr FW, Morice MC, Kappetein AP, et al. Coronary artery bypass graft surgery versus percutaneous coronary intervention in patients with three-vessel disease and left main coronary disease: 5-year follow-up of the randomised, clinical SYNTAX trial. Lancet 2013; 381: 629-38.

11. Puri R, Kapadia SR, Nicholls SJ, et al. Optimizing outcomes during left main percutaneous coronary intervention with intravascular ultrasound and fractional flow reserve: the current state of evidence. JACC Cardiovasc Interv 2012; 5: 697-707.

12. Lee MS, Kapoor N, Jamal F, et al. Comparison of coronary artery bypass surgery with percutaneous coronary intervention with drug-eluting stents for unprotected left main coronary artery disease. J Am Coll Cardiol 2006; 47: 864-70.

13. Colombo A, Bramucci E, Sacca S, et al. Randomized study of the crush technique versus provisional side-branch stenting in true coronary bifurcations: the CACTUS (Coronary Bifurcations: Application of the Crushing Technique Using Sirolimus-Eluting Stents) Study. Circulation 2009; 119: 71-8.

14. Verheye S, Ramcharitar S, Grube E, et al. Six-month clinical and angiographic results of the STENTYS(R) self-apposing stent in bifurcation lesions. Eurolntervention 2011; 7: 580-7.

15. Laborde JC, Borenstein N, Behr L, Ramcharitar S. Stentys coronary bifurcation stent. Eurolntervention 2007; 3: 162-5.

16. Park SJ, Kim YH, Park DW, et al. Impact of intravascular ultrasound guidance on long-term mortality in stenting for unprotected left main coronary artery stenosis. Circulation Cardiovasc Interv 2009; 2: 167-77. 